Case Report

\title{
Acute Gastrointestinal Bleeding in Olmesartan-Associated Collagenous Gastroduodenitis: A Potential Endoscopic Complication
}

\author{
Rachel Hudacko $\mathbb{D}^{1}$ and Lance Siegel ${ }^{2}$ \\ ${ }^{1}$ Orange Pathology Associates, PC, Department of Pathology, Orange Regional Medical Center, 707 East Main St., \\ Middletown, NY 10940, USA \\ ${ }^{2}$ Horizon Family Medical Group, Department of Gastroenterology, Orange Regional Medical Center, 707 East Main St., \\ Middletown, NY 10940, USA \\ Correspondence should be addressed to Rachel Hudacko; rhudacko@gmail.com
}

Received 3 November 2017; Accepted 12 February 2018; Published 14 March 2018

Academic Editor: Chia-Tung Shun

Copyright (C) 2018 Rachel Hudacko and Lance Siegel. This is an open access article distributed under the Creative Commons Attribution License, which permits unrestricted use, distribution, and reproduction in any medium, provided the original work is properly cited.

\begin{abstract}
Collagenous gastroenteritis is a rare disease that is known to be associated with the drug olmesartan, an angiotensin II receptor antagonist used to treat hypertension. It is characterized histologically by increased subepithelial collagen deposition with associated inflammation and epithelial injury. Endoscopically, the mucosa appears inflamed and friable and may be nodular or atrophic. We report a case of acute gastric bleeding on direct mucosal contact during endoscopy in a patient with olmesartan-associated collagenous gastroduodenitis to raise awareness of this potential endoscopic complication.
\end{abstract}

\section{Introduction}

Collagenous gastroenteritis is a pattern of injury that is characterized histologically by subepithelial collagen deposition $>10 \mu \mathrm{m}$ in thickness with associated mucosal inflammation and epithelial injury [1]. The collagen bands are usually irregular, often entrap small capillaries, and can be highlighted with a trichrome stain. It is a rare disorder that may involve the stomach (collagenous gastritis) and/or small bowel (collagenous enteritis/sprue) and may occasionally coexist with the more common entity, collagenous colitis. The etiology of collagenous gastroenteritis is unknown but is suspected to be immune-mediated and may be associated with certain medications including the antihypertensive drug olmesartan, an angiotensin II receptor antagonist [2]. Several reports in the literature describe cases of olmesartanassociated collagenous duodenitis/sprue [3-5] with fewer reports of olmesartan-associated collagenous gastritis $[3,6]$ and only rare reports involving both the stomach and small bowel [3]. We herein report a case of olmesartan-associated collagenous gastroduodenitis that was complicated by acute gastrointestinal bleeding during endoscopy.

\section{Case Presentation}

A 64-year-old woman presented to the gastroenterologist with abdominal pain, unintentional 10 pound weight loss over 3 months, and reflux symptoms. Pertinent medical history included hypertension treated with olmesartan $20 \mathrm{mg}$ once a day for the past 7 years, hyperlipidemia treated with simvastatin, gastroesophageal reflux disease treated with pantoprazole $20 \mathrm{mg}$ twice a day, and osteoporosis treated with denosumab injections every 6 months. Esophagogastroduodenoscopy (EGD) performed in the office showed granular mucosa in the proximal duodenum and nodular mucosa in the stomach with numerous linear ulcers. On withdrawal of the scope from the duodenum, a large amount of fresh blood was noted in the stomach with clotted blood near the cardia. The bleeding was believed to be due to either the underlying gastric ulcers or a Mallory Weiss tear. The patient 


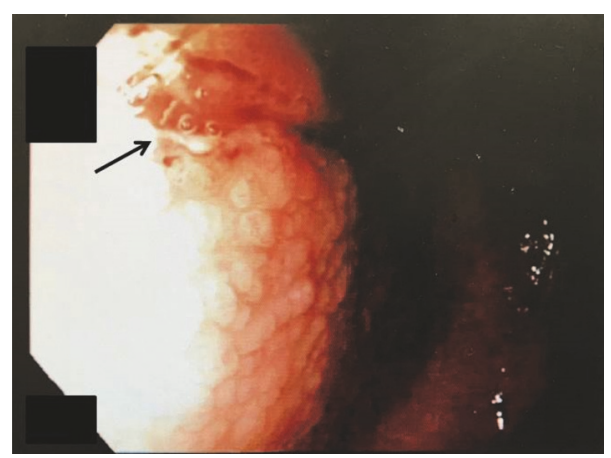

(a)

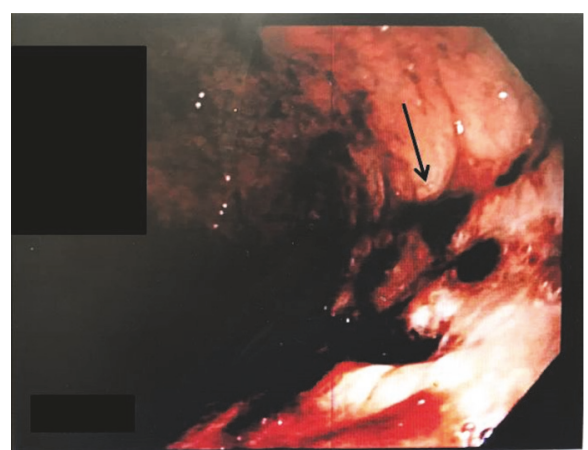

(b)

FIGURE 1: Endoscopic photos of the body of the stomach show diffusely nodular mucosa (a) with deep linear ulcers (arrows) and severe inflammation and hemorrhage (b).

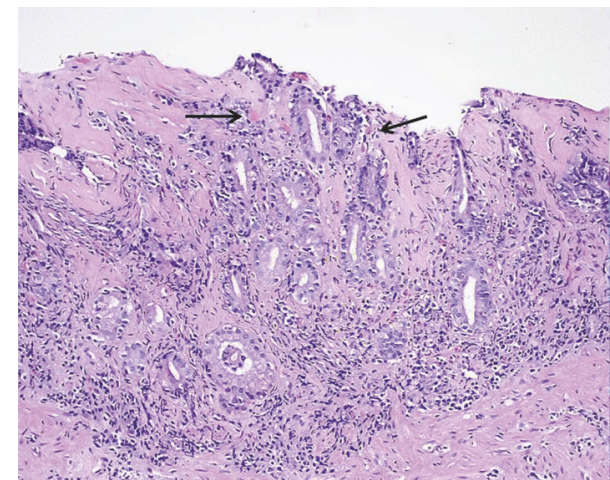

(a)

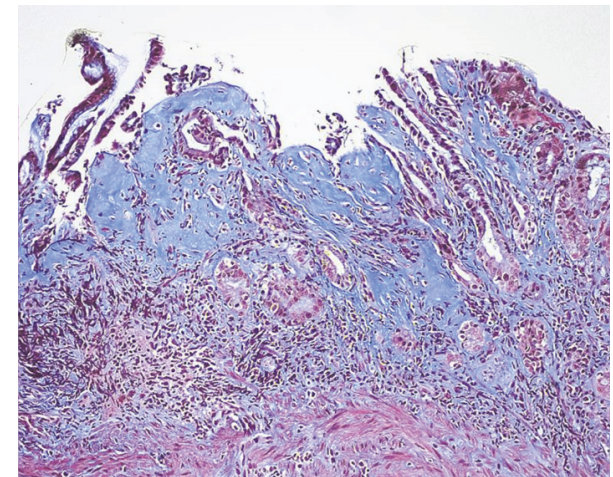

(b)

FIGURE 2: 20x magnification: (a) hematoxylin and eosin-stained section of the gastric body shows a thick, markedly irregular pink band of subepithelial collagen deposition with complete surface erosion. The mucosa is inflamed and atrophic with loss of parietal cells. Arrows indicate entrapped capillaries. (b) Trichrome stain highlights the collagen blue.

was transferred to the emergency department for further evaluation.

On admission, the hemoglobin and hematocrit were normal, and an emergent EGD was performed. The gastric fundus and body appeared atrophic with friable and severely inflamed mucosa and multiple linear ulcers without active bleeding (Figure 1). The antral mucosa appeared erythematous. Biopsies of the stomach and duodenum were performed. The biopsies of the antrum and body showed increased subepithelial collagen deposition confirmed on a trichrome stain, chronic inflammation in the lamina propria, surface epithelial regenerative changes, and erosions, consistent with collagenous gastritis (Figure 2). The gastric body mucosa was atrophic with near-total loss of parietal cells. Immunostain for $H$. pylori organisms was negative, and a Congo red stain for amyloid was negative. The biopsies of the duodenum showed partial villous atrophy and increased subepithelial collagen deposition confirmed on a trichrome stain, consistent with collagenous duodenitis/sprue (Figure 3). The patient was started on high dose acid suppression with pantoprazole $40 \mathrm{mg}$ twice a day, and the olmesartan was discontinued.
Follow-up EGD 7 weeks later showed somewhat atrophic gastric mucosa with few residual linear areas of erythema and complete resolution of the ulcers, inflammation, and mucosal friability (Figure 4). The duodenal mucosa appeared normal. Colonoscopy was unremarkable. Repeat biopsies showed marked improvement of the collagenous gastritis with resolution of the epithelial injury and only rare residual foci of subepithelial collagen. A mild nonspecific chronic inactive gastritis remained present. The biopsies from the duodenum and left colon showed normal mucosa without evidence of collagenous duodenitis or collagenous colitis.

\section{Brief Discussion}

Olmesartan-associated collagenous gastroenteritis is a rare entity that may be immune-mediated in nature. The length of time of olmesartan use before onset of symptoms varies from less than 1 month to 11 years [7]. When the stomach is solely involved, symptoms include dyspepsia, anemia due to bleeding, weight loss, and diarrhea. When the small intestine is involved, the most common symptoms are watery diarrhea and weight loss due to malabsorption [2]. The 


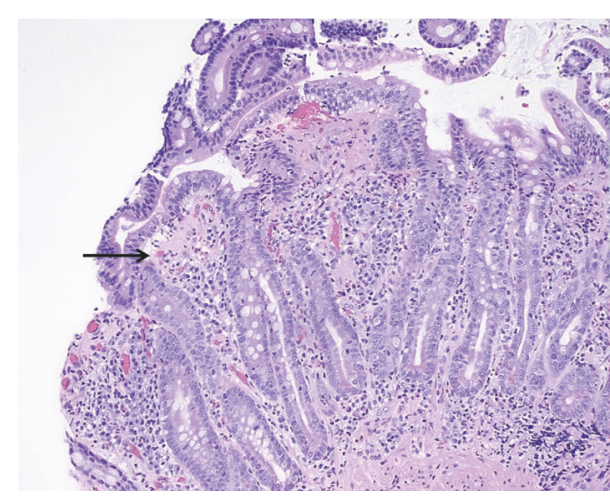

(a)

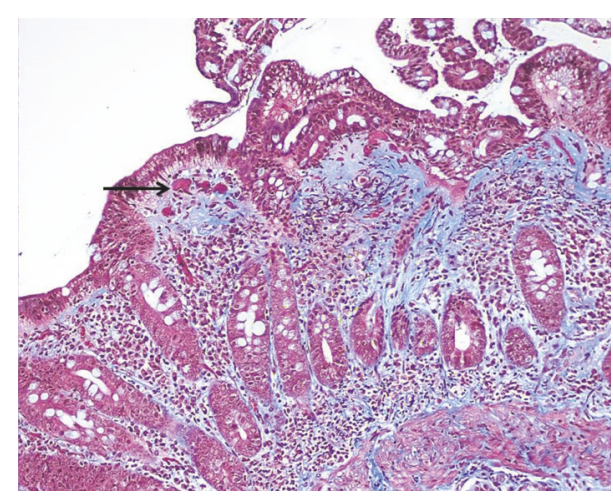

(b)

FIGURE 3: 20x magnification: (a) hematoxylin and eosin-stained section of the duodenum shows villous shortening/partial atrophy with increased subepithelial collagen deposition (pink). (b) Trichrome stain highlights the collagen blue. Arrows indicate entrapped capillaries.
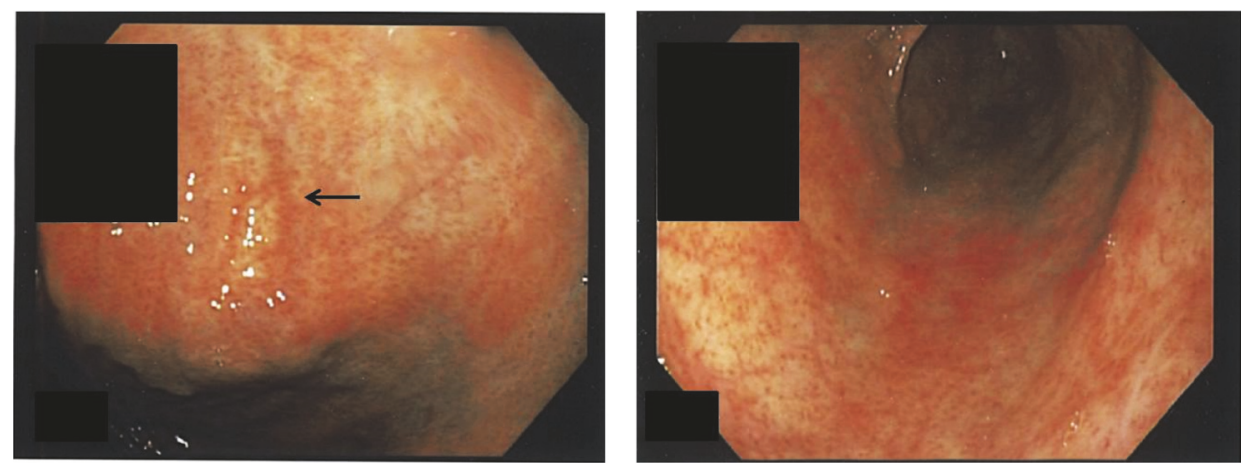

FIGURE 4: Endoscopic photos of the body of the stomach 7 weeks after cessation of olmesartan show atrophic mucosa with few residual areas of erythema (arrow) and complete resolution of the ulcers.

characteristic endoscopic finding of collagenous gastritis is nodular mucosa. Other findings include erythema, mucosal friability, erosions, ulcers, and atrophy $[2,6]$. The endoscopic findings in the small bowel are nonspecific and include pale mucosa, mucosal thickening, and scalloping $[2,4]$.

The diagnostic histologic feature of collagenous gastroenteritis is band-like layer of subepithelial collagen deposition that is at least $10 \mu \mathrm{m}$ in thickness and is associated with a chronic inflammatory infiltrate and surface epithelial injury [1]. The deposits can be patchy and irregular and often entrap superficial dilated capillaries [2]. Bleeding results from surface epithelial injury and sloughing which exposes these capillaries.

The majority of cases of olmesartan-associated collagenous gastroenteritis reported in the literature resolved after cessation of the drug [7]. One study reported complete clinical and pathologic resolution of collagenous duodenitis after treatment with the immunosuppressive drugs FK-506 and rapamycin [8]. Our patient had a complete clinical response and near-complete pathologic response at 7-week follow-up after discontinuation of olmesartan.

In summary, we report a case of acute gastric bleeding during endoscopy due to olmesartan-associated collagenous gastroduodenitis. The mechanism of bleeding in this disease is the presence of abnormal subepithelial collagen causing mucosal fragility. Bleeding may be spontaneous and chronic with associated anemia or may be acute and caused by direct contact with instruments during endoscopy, as was believed to be the reason for the acute bleed in this case. Although rare, it is important for endoscopists to be aware of this potential endoscopic complication in patients taking olmesartan.

\section{Conflicts of Interest}

There are no conflicts of interest or funding sources.

\section{References}

[1] R. B. Colletti and T. D. Trainer, "Collagenous gastritis," Gastroenterology, vol. 97, no. 6, pp. 1552-1555, 1989.

[2] O. H. Nielsen, L. B. Riis, S. Danese, R. D. Bojesen, and C. Soendergaard, "Proximal collagenous gastroenteritides: clinical management: a systematic review," Annals of Medicine, vol. 46, no. 5, pp. 311-317, 2014.

[3] A. Rubio-Tapia, M. L. Herman, J. F. Ludvigsson et al., "Severe spruelike enteropathy associated with olmesartan," Mayo Clinic Proceedings, vol. 87, no. 8, pp. 732-738, 2012. 
[4] J. A. Nielsen, A. Steephen, and M. Lewin, "Angiotensin-II inhibitor (olmesartan)-induced collagenous sprue with resolution following discontinuation of drug," World Journal of Gastroenterology, vol. 19, no. 40, pp. 6928-6930, 2013.

[5] C. Desruisseaux, M. Bensoussan, E. Désilets et al., "Adding water to the mill: olmesartan-induced collagenous sprue-a case report and brief literature review," Canadian Journal of Gastroenterology and Hepatology, vol. 2016, Article ID 4837270, 2 pages, 2016.

[6] C. Ma, J. Y. Park, E. A. Montgomery et al., "A comparative clinicopathologic study of collagenous gastritis in children and adults: The same disorder with associated immune-mediated diseases," The American Journal of Surgical Pathology, vol. 39, no. 6, pp. 802-812, 2015.

[7] N. Burbure, B. Lebwohl, C. Arguelles-Grande, P. H. R. Green, G. Bhagat, and S. Lagana, "Olmesartan-associated sprue-like enteropathy: A systematic review with emphasis on histopathology," Human Pathology, vol. 50, pp. 127-134, 2016.

[8] S. Scialom, G. Malamut, B. Meresse et al., "Gastrointestinal disorder associated with olmesartan mimics autoimmune enteropathy," PLoS ONE, vol. 10, no. 6, article e0125024, 2015. 


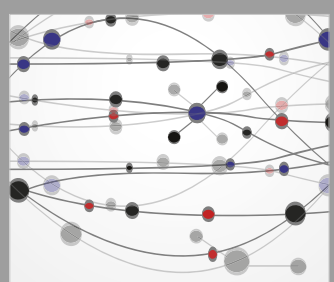

The Scientific World Journal
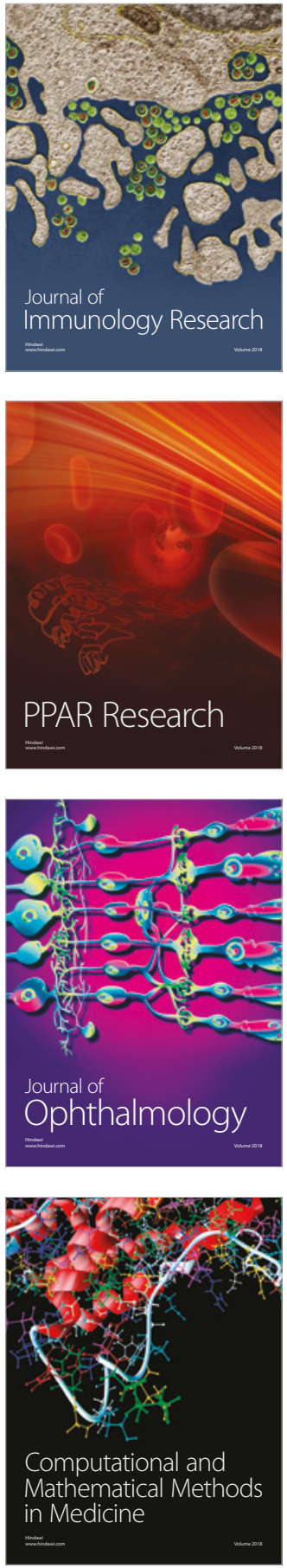

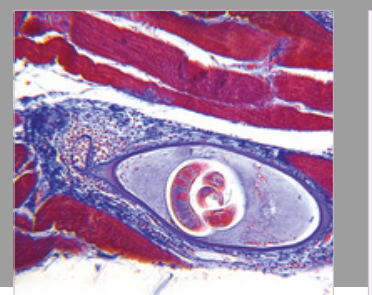

Gastroenterology Research and Practice

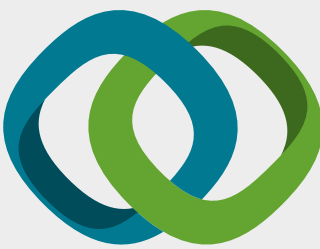

\section{Hindawi}

Submit your manuscripts at

www.hindawi.com
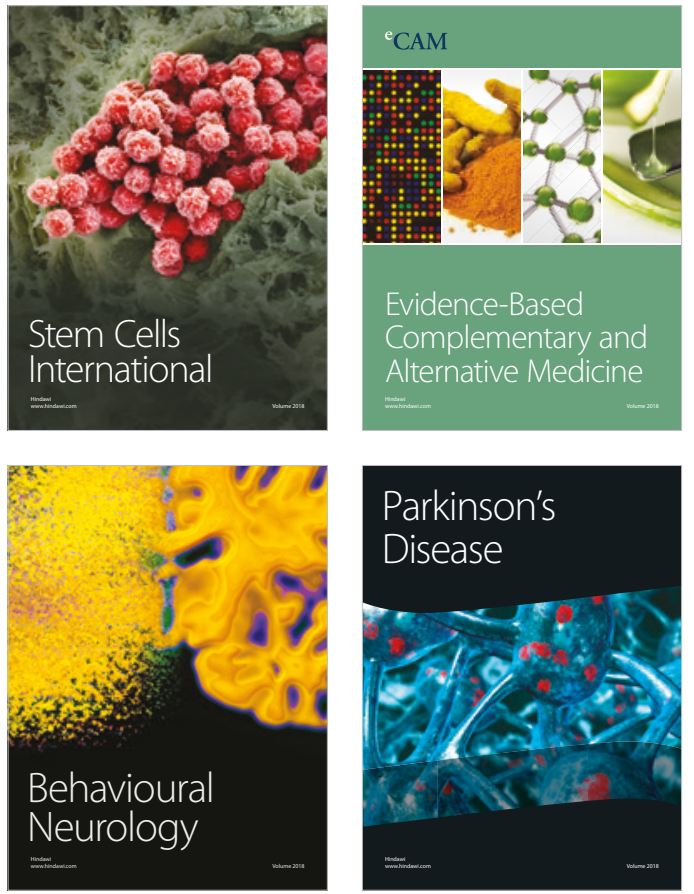

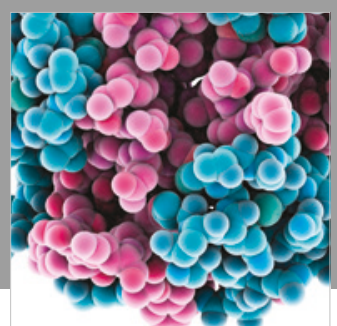

ournal of

Diabetes Research

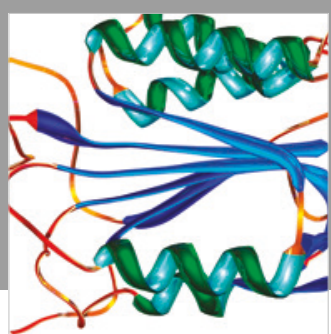

Disease Markers
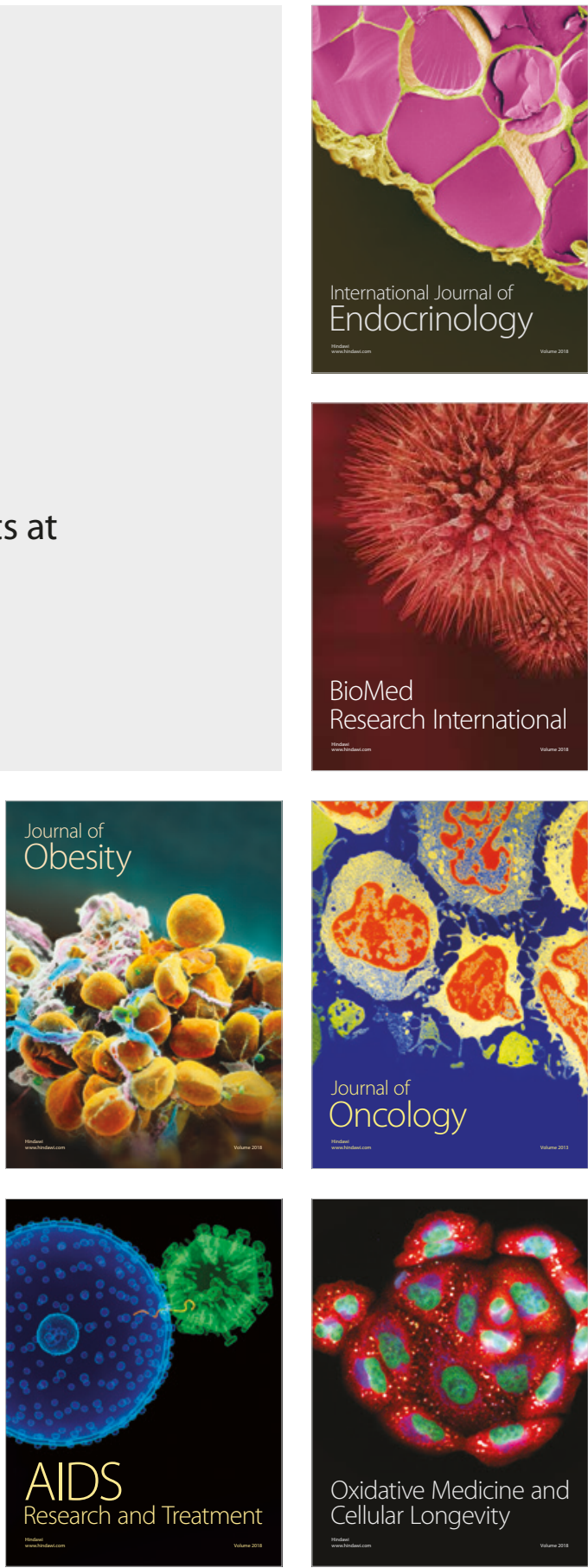